\title{
Tarski 模型外的一类机器可判定问题
}

\section{杨 路 1,2 姚 勇 ${ }^{1 *}$ 冯 勇 ${ }^{1}$}

(1. 中国科学院成都计算机应用研究所, 成都 $610041 ， 2$. 华东师范大学计算机理论研究所, 上海 200062)

摘要利用对称多项式的降维方法和证明代数不等式的胞腔分解方法, 给出了一个实用的 算法, 用于判定一类变元个数也是变量的多项式正性命题. 这是一类在 Tarski 模型外的机器 可判定问题. 在 Maple 平台上, 根据该算法设计的程序 nprove, 可以快速实现判定目标.

关键词 对称多项式不等式 胞腔分解 Tarski 模型 机器可判定问题 $\operatorname{MSC}(2000)$ 主题分类 $68 \mathrm{~T} 15,03 \mathrm{~B} 35$

\section{1 引言}

20 世纪 50 年代初, 波兰数学家 Tarski ${ }^{[1]}$ 发表了著名的论文 《初等代数与初等几何的判 定问题》, 证明了初等代数以及初等几何范围的命题, 可以用机械的步骤来判定其对与否, 此 种问题称为是机器 (或算法) 可判定的. 这里所说的初等代数范围, 是指相关命题都可在满足 实闭域公理的前提下, 表达为由常量符号 $\{0,1\}$, 变量符号 $\left\{x_{1}, \ldots, x_{k}\right\}$, 运算符号 $\{+,-, \times\}$, 关系符号 $\{=,<\}$ 和逻辑关联词所构成的一阶语句, 这里任意一个确定的公式中变元个数都 是确定的有限数. 由于 Tarski 的杰出贡献, 这类语句通常统称为 Tarski 模型. Tarski 模型中 的命题是机器可判定的, 但是另一方面由 Gödel 的著名定理, 机器可判定的问题类在数学中 相对较少, 即使看来最简单的初等数论这一范围, 其中命题的机器判定整体而言也是不可能 实现的. 尽管如此, 在已经过去的 30 年间, 定理机器证明领域仍变得非常繁荣, 一系列的新

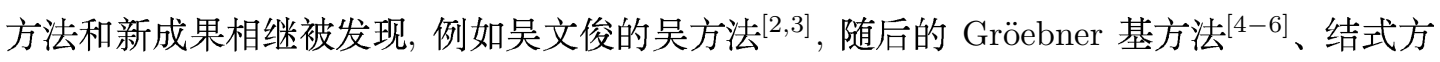
法 ${ }^{[7]}$ 、数值并行法 ${ }^{[8]}$, 能产生可读证明的消点法 ${ }^{[9]}$, 以及由 Collins 提出并经后人改进的柱 形代数分解方法 ${ }^{[10]}$, 等等, 不过这些方法所处理的问题类基本属于 Tarski 模型, 所以研讨 Tarski 模型外且具有实际应用价值的机器可判定问题类是极有意义的事, 本文即将讨论这样 的一个问题类. 为了使说明更为生动, 先举如下一个具体问题的例:

问题 1 具有 $n$ 个变元的多项式

$$
\begin{aligned}
f= & -\left(\sum_{k=1}^{n} x_{k}^{5}\right)-6\left(\sum_{k=1}^{n} x_{k}^{4}\right)\left(\sum_{k=1}^{n} x_{k}\right) \\
& +2\left(\sum_{k=1}^{n} x_{k}^{3}\right)\left(\sum_{k=1}^{n} x_{k}^{2}\right)+8\left(\sum_{k=1}^{n} x_{k}^{3}\right)\left(\sum_{k=1}^{n} x_{k}\right)^{2}
\end{aligned}
$$

收稿日期: 2006-11-16; 接受日期: 2007-02-28 


$$
+3\left(\sum_{k=1}^{n} x_{k}^{2}\right)^{2}\left(\sum_{k=1}^{n} x_{k}\right)-6\left(\sum_{k=1}^{n} x_{k}^{2}\right)\left(\sum_{k=1}^{n} x_{k}\right)^{3}+\left(\sum_{k=1}^{n} x_{k}\right)^{5},
$$

其中 $x_{i} \in \mathbb{R}_{+}, i=1, \ldots, n$, 是否对一切自然数 $n$ 均有 $f \geqslant 0$ 成立?

这个问题有一个很显著的特征, 即 $f$ 的变元个数 $n$ 是变化的不确定的自然数, 因而此问 题不在 Tarski 模型内. 就一般而言, 涉及变元个数也是变量的问题类仍是机器不可判定的, 但 是我们成功的在其中找到了一个子类是机器可判定的, 并得到了有效的算法, 已编程在机器 上实现. 这有点类似于代数方程根式解问题, 一般的代数方程根据 Abel 定理是没有根式解的, 但其中有一些子方程类是可根式解的.

\section{2 问题的描述与预备知识}

本文中研究的问题是

问题 2 下述命题是否是机器可判定的?

“对任意给定的有理系数齐次对称多项式 $f\left(x_{1}, x_{2}, \ldots, x_{n}\right)$, 不等式 $f\left(x_{1}, x_{2}, \ldots, x_{n}\right) \geqslant 0$ 对一切自然数 $n$ 均成立, 其中 $x_{i} \in \mathbb{R}_{+}, i=1, \ldots, n$ ”.

为了讨论问题 2 , 我们需要对称多项式的一些熟知基本结果和一个最近出现的新结果.

定义 2.1 齐次多项式 $f\left(x_{1}, x_{2}, \ldots, x_{n}\right)$ 称为对称的, 如果

$$
f\left(x_{1}, x_{2}, \ldots, x_{n}\right)=f\left(\sigma\left(x_{1}, x_{2}, \ldots, x_{n}\right)\right)
$$

对所有的 $\sigma \in S_{n}$ 成立, 这里 $S_{n}$ 是 $n$ 个文字的全对称置换群.

齐次多项式也简称型, 记实数域上 $n$ 元 $m$ 次对称型所成集合为 $S_{n, m}$, 在通常加法和数 乘下它形成实向量空间, 维数记为 $\operatorname{dim}\left(S_{n, m}\right)$.

定义 2.2 对正整数 $k$, 一个固定点 $x=\left(x_{1}, x_{2}, \ldots, x_{n}\right) \in \mathbb{R}^{n}$ 的 $k$ 次幂和定义为

$$
P_{(n, k)}(x)=\sum_{j=1}^{n} x_{j}^{k} .
$$

引理 2.1 (对称型基本定理) 对称型 $f \in S_{n, m}$ 可以唯一的表示为 $P_{(n, 1)}, P_{(n, 2)}, \ldots, P_{(n, d)}$ 的多项式, 这里 $d=\min (n, m)$. 并且 $P_{(n, 1)}, P_{(n, 2)}, \ldots, P_{(n, d)}$ 是代数无关的, 即不存在非 0 多 项式 $g$, 使得 $g\left(P_{(n, 1)}, P_{(n, 2)}, \ldots, P_{(n, d)}\right)=0$.

记不定方程

$$
1 y_{1}+2 y_{2}+\cdots+d y_{d}=m
$$

的非负整数解集为 $\Omega$.

引理 2.2 集合 $B_{n, m}=\left\{P_{(n, 1)}^{\lambda_{1}} P_{(n, 2)}^{\lambda_{2}} \cdots P_{(n, d)}^{\lambda_{d}} \mid\left(\lambda_{1}, \lambda_{2}, \ldots, \lambda_{d}\right) \in \Omega\right\}$ 是向量空间 $S_{n, m}$ 的 一组基, $S_{n, m}$ 的维数是集合 $\Omega$ 中元素个数, 其中 $d=\min (n, m)$.

按照引理 2.2 , 齐 5 次对称多项式可以表为

$$
\begin{aligned}
g= & a P_{(n, 5)}+b P_{(n, 4)} P_{(n, 1)}+c P_{(n, 3)} P_{(n, 2)}+d P_{(n, 3)} P_{(n, 1)}^{2}+e P_{(n, 2)}^{2} P_{(n, 1)} \\
& +\alpha P_{(n, 2)} P_{(n, 1)}^{3}+\beta P_{(n, 1)}^{5} \\
= & (n,[a, b, c, d, e, \alpha, \beta]) .
\end{aligned}
$$


问题 1 中出现的多项式 $f$, 按简记形式 (3) 也就是 $f=(n,[-1,-6,2,8,3,-6,1])$. 将对称 多项式表示成幂和的形式, 优点是立即可以推广到 $n$ 变元的情形.

我们还需要用到对称多项式半正定性判定的一个最新结果. 为了较严格的来叙述这个结 果, 先解释几个常用记号的含义:

$$
1_{k}=(\underbrace{1,1, \ldots, 1}_{k}) ; \quad 0_{k}=(\underbrace{0,0, \ldots, 0}_{k}) ;
$$

$[d]$ 表示不超过 $d$ 的最大整数;

对任意的 $x=\left(x_{1}, x_{2}, \ldots, x_{n}\right) \in \mathbb{R}^{n}$, 记

$$
\begin{aligned}
& v(x)=\left|\left\{x_{j} \mid j=1,2, \ldots, n\right\}\right|, \\
& v(x)^{*}=\left|\left\{x_{j} \mid x_{j} \neq 0, j=1,2, \ldots, n\right\}\right| ;
\end{aligned}
$$

此处 $|A|$ 表示集合 $A$ 中元素个数. $v(x)$ 的一个直观的解释是, 点 $x$ 的坐标中互不相同的数的 个数, $v(x)^{*}$ 类似理解.

引理 $2.3^{[11,12]}$ (i) 一个 $n$ 元 $d$ 次对称型不等式在 $\mathbb{R}_{+}^{n}$ 上成立, 当且仅当这个不等式在 集合 $\left\{x \mid x \in \mathbb{R}_{+}^{n}, v(x)^{*} \leqslant \max \left(\left[\frac{d}{2}\right], 1\right)\right\}$ 上是成立的.

(ii) 一个 $n$ 元 $d$ 次对称型不等式在 $\mathbb{R}^{n}$ 上成立, 当且仅当这个不等式在集合 $\{x \mid x \in$ $\left.\mathbb{R}^{n}, v(x) \leqslant \max \left(\left[\frac{d}{2}\right], 2\right)\right\}$ 上是成立的.

引理 2.3 的意义在于将多变元多项式的问题化为一系列更少变元数的问题, 起到了降低 维数的作用.

当取 $d=3$ 时, 就可以得到如下著名的结果:

引理 2.4 ${ }^{[13]} f \in S_{n, 3}$, 在 $\mathbb{R}_{+}^{n}$ 上 $f \geqslant 0$ 的充分必要条件是: $f\left(1_{k}, 0_{n-k}\right) \geqslant 0$ 对每一个 $k=1,2, \ldots, n$ 成立.

这样在证明一个 3 次的对称型不等式的时候, 只需对 $n$ 个点 $\left(1_{k}, 0_{n-k}\right)(k=1,2, \ldots, n)$ 作验证就可以了. 对 4 和 5 次对称形式不等式有如下的结果:

引理 2.5 对任意的 $f \in S_{n, p}$, 其中 $p \in\{4,5\}$, 有下面等价关系成立:

$$
f(x) \geqslant 0, x \in \mathbb{R}_{+}^{n} \Longleftrightarrow f\left(t \cdot 1_{r}, 1_{s}, 0_{n-r-s}\right) \geqslant 0, \quad \forall t \in \mathbb{R}_{+}, \quad \forall(r, s) \in \mathbb{N}_{n}
$$

$\mathbb{N}_{n}=\{(r, s) \mid r, s$ 都是正整数, 且 $r+s \leqslant n\}$.

证明 由引理 2.3 可得

$$
f(x) \geqslant 0, x \in \mathbb{R}_{+}^{n} \Longleftrightarrow f(y) \geqslant 0, \quad \forall y \in \mathbb{R}_{+}^{n}, \quad v^{*}(y) \leqslant 2 .
$$

因 $f$ 是对称形式, 故可以令 $y=\left(t_{1} \cdot 1_{r}, t_{2} \cdot 1_{s}, 0_{n-r-s}\right), t_{1}, t_{2} \in \mathbb{R}_{+}$. 注意到 $f$ 又是齐次多 项式, 即有

$$
\begin{aligned}
f(y) \geqslant 0, \quad \forall y \in \mathbb{R}_{+}^{n}, \quad v^{*}(y) \leqslant 2 & \Longleftrightarrow\left(t_{2}\right)^{p} f\left(\frac{t_{1}}{t_{2}} \cdot 1_{r}, 1_{s}, 0_{n-r-s}\right) \geqslant 0, \quad \forall t_{1}, t_{2} \in \mathbb{R}_{+}, \quad t_{2} \neq 0, \quad \forall(r, s) \in \mathbb{N}_{n} \\
& \Longleftrightarrow f\left(\frac{t_{1}}{t_{2}} \cdot 1_{r}, 1_{s}, 0_{n-r-s}\right) \geqslant 0, \quad \forall t_{1}, t_{2} \in \mathbb{R}_{+}, \quad t_{2} \neq 0, \quad \forall(r, s) \in \mathbb{N}_{n} \\
& \Longleftrightarrow f\left(t \cdot 1_{r}, 1_{s}, 0_{n-r-s}\right) \geqslant 0, \quad \forall t \in \mathbb{R}_{+}, \quad \forall(r, s) \in \mathbb{N}_{n}
\end{aligned}
$$


证毕.

如果记

$$
\begin{aligned}
f_{r, s}(t)= & a\left(r t^{5}+s\right)+b\left(r t^{4}+s\right)(r t+s) \\
& +c\left(r t^{3}+s\right)\left(r t^{2}+s\right)+d\left(r t^{3}+s\right)(r t+s)^{2}+e\left(r t^{2}+s\right)^{2}(r t+s) \\
& +\alpha\left(r t^{2}+s\right)(r t+s)^{3}+\beta(r t+s)^{5}
\end{aligned}
$$

展开整理为 $t$ 的多项式

$$
\begin{aligned}
& f_{r, s}(t)=A_{r, s} t^{5}+B_{r, s} t^{4}+C_{r, s} t^{3}+D_{r, s} t^{2}+E_{r, s} t+H_{r, s}, \\
& \left\{\begin{array}{l}
A_{r, s}=r\left(\beta r^{4}+\alpha r^{3}+(d+e) r^{2}+(b+c) r+a\right), \\
B_{r, s}=s r\left(5 \beta r^{3}+3 \alpha r^{2}+(2 d+e) r+b\right), \\
C_{r, s}=s r\left((\alpha+10 s \beta) r^{2}+(3 \alpha s+2 e) r+d s+c\right), \\
D_{r, s}=s r\left((\alpha+10 r \beta) s^{2}+(3 \alpha r+2 e) s+d r+c\right), \\
E_{r, s}=s r\left(5 \beta s^{3}+3 \alpha s^{2}+(2 d+e) s+b\right), \\
H_{r, s}=s\left(\beta s^{4}+\alpha s^{3}+(d+e) s^{2}+(b+c) s+a\right) .
\end{array}\right.
\end{aligned}
$$

由公式 $(2)$ 可见 $f_{r, s}(t)$ 的直观意义是: 5 次对称型 $f\left(x_{1}, x_{2}, \ldots, x_{n}\right)$ 中有 $r$ 个变元取 $t$, 有 $s$ 个取 1 , 剩余的取 0 时的值.

由引理 2.5 , 我们得到

引理 $2.6 f\left(x_{1}, x_{2}, \ldots, x_{n}\right) \in S_{n, 5},\left(x_{1}, x_{2}, \ldots, x_{n}\right) \in \mathbb{R}_{+}^{n}$, 则 $f\left(x_{1}, x_{2}, \ldots, x_{n}\right) \geqslant 0$ 对一 切自然数 $n$ 均成立的充分必要条件是: 对任意自然数 $r$ 和 $s$, 以及 $\forall t \in \mathbb{R}_{+}$, 有 $f_{r, s}(t) \geqslant 0$.

有了上面的这些准备工作, 下面就可以给出本文的主要结果了.

\section{3 主要结果的证明与算法描述}

我们的主要结果是

定理 1 下述命题是机器可判定的问题类:

“对任意给定的次数不超过 5 的有理系数对称型 $g\left(x_{1}, x_{2}, \ldots, x_{n}\right)$, 其中 $\left(x_{1}, x_{2}, \ldots, x_{n}\right) \in$ $\mathbb{R}_{+}^{n}$, 不等式 $g\left(x_{1}, x_{2}, \ldots, x_{n}\right) \geqslant 0$ 对一切自然数 $n$ 均成立”.

我们对定理 1 的证明采用直接法, 即直接构造算法, 再证明算法的正确性和可终止性. 一 般情形下引理 2.6 并不能直接用来证明定理 1 , 原因在于需要对数目不确定的自然数偶 $(r, s)$ 作检测, 这是机器所没法做到的, 所以需要做些改造. 我们的基本思想是: 将完全离散的点集 $(r, s)$, 改造为部分离散部分连续形式, 将问题化归到 Tarski 模型内来解决. 为了不使证明的 思路被细枝末节淹没掉, 我们将略去一些不重要的细节, 比如只对 5 次对称型给出证明, 5 次 以下的对称型显然可以通过乘上一个对称因子 $\left(x_{1}+\cdots+x_{n}\right)^{5-i}$ 变为 5 次的.

引理 $3.1 f\left(x_{1}, x_{2}, \ldots, x_{n}\right)=(n,[a, b, c, d, e, \alpha, \beta]) \in S_{n, 5}$, 其中 $\left(x_{1}, x_{2}, \ldots, x_{n}\right) \in \mathbb{R}_{+}^{n}$. 若 
不等式 $f\left(x_{1}, x_{2}, \ldots, x_{n}\right) \geqslant 0$ 对一切自然数 $n$ 均成立, 则

$$
\begin{aligned}
& \beta>0 \text { 或 } \\
& \beta=0, \alpha>0 \text { 或 } \\
& \beta=\alpha=0, d+e>0, d \geqslant 0 \text { 或 } \\
& \beta=\alpha=d+e=0, d>0, b+c>0 \text { 或 } \\
& \beta=\alpha=d=e=0, b+c>0, b \geqslant 0 \text { 或 } \\
& \beta=\alpha=d=e=b+c=0, b>0 \text { 或 } \\
& \beta=\alpha=d=e=b=c=0, a \geqslant 0 .
\end{aligned}
$$

证明 根据已知, 由引理 2.6 , 对任意自然数 $r$ 和 $s$, 以及 $\forall t \in \mathbb{R}_{+}$, 均有 $f_{r, s}(t) \geqslant 0$, 特别 对任意自然数 $s$, 有 $f_{r, s}(0) \geqslant 0$, 即 $H_{r, s} \geqslant 0$. 当 $s$ 取充分大的自然数时, 熟知 $H_{r, s}$ 的符号由 它关于 $s$ 的最高次项系数决定, 即 $s$ 充分大时 $H_{r, s}$ 与 $\beta$ 同符号, 故 $\beta \geqslant 0$.

若 $\beta=0$, 完全类似可证得 $\alpha \geqslant 0$.

若 $\beta=\alpha=0$, 也类似可证得 $d+e \geqslant 0$, 下证此时还有 $d \geqslant 0$, 在 (5) 式中取 $r=1, s=t^{2}$, 这时

$$
f_{r, s}(t)=(t+1) t^{2}\left(d t^{4}+(2 d+b+4 e) t^{3}+(a+2 c+d) t^{2}+(b-a) t+a\right)
$$

可见当 $t$ 充分大时 $f_{r, s}(t)$ 的值的符号与 $d$ 的符号相同, 故 $d \geqslant 0$.

剩下的情形依次类似讨论即可.

引理 $3.2^{[14]}$ 设 $x_{0}$ 是实系数多项式

$$
g(x)=x^{n}+a_{n-1} x^{n-1}+\cdots+a_{0}
$$

的任一实根, 则 $x_{0} \leqslant 1+\max \left\{\left|a_{0}\right|, \ldots,\left|a_{n-1}\right|\right\}$.

引理 3.2 意味着当 $x>1+\max \left\{\left|a_{0}\right|, \ldots,\left|a_{n-1}\right|\right\}$ 时 $g(x)>0$, 因为 $g(x)=0$, 此时已经没 有实根了.

引理 $3.3 f\left(x_{1}, x_{2}, \ldots, x_{n}\right)=(n,[a, b, c, d, e, \alpha, \beta]) \in S_{n, 5}$, 其中 $\left(x_{1}, x_{2}, \ldots, x_{n}\right) \in \mathbb{R}_{+}^{n}$. 若不等式 $f\left(x_{1}, x_{2}, \ldots, x_{n}\right) \geqslant 0$ 对一切自然数 $n$ 均成立, 则必存在实数 $r_{0}$ 和 $s_{0}$, 当 $r$ 和 $s$ 为 实数且 $r>r_{0}, s>s_{0}, t \in \mathbb{R}_{+}$时有 $f_{r, s}(t) \geqslant 0$.

证明 (I) 首先讨论 $[\beta>0]$ 或 $[\beta=0, \alpha>0]$ 的情形.

由条件 $[\beta>0]$ 或 $[\beta=0, \alpha>0]$, 从引理 3.2 知, 存在正实数 $r_{1}$ 和 $s_{1}$ 当 $r>r_{1}, s>s_{1}$ 时 (7) 式中的 $A_{r, s} \geqslant 0, B_{r, s} \geqslant 0, E_{r, s} \geqslant 0, H_{r, s} \geqslant 0$.

从 $C_{r, s}$ 和 $D_{r, s}$ 的表达式可见 $r$ 和 $s$ 地位对等, 故不妨设 $r \geqslant s$, 令 $r=s+y\left(y \in \mathbb{R}_{+}\right)$代 入 $C_{r, s}$ 和 $D_{r, s}$ 整理为 $y$ 的多项式

$$
\begin{aligned}
& C_{r, s}=(10 \beta s+\alpha) y^{2}+\left(20 \beta s^{2}+5 \alpha s+2 e\right) y+10 \beta s^{3}+4 \alpha s^{2}+(d+2 e) s+c, \\
& D_{r, s}=\left(10 \beta s^{2}+3 \alpha s+d\right) y+10 \beta s^{3}+4 \alpha s^{2}+(d+2 e) s+c .
\end{aligned}
$$


再从引理 3.2 知, 存在正实数 $s_{2}$, 当 $s>s_{2}$ 时,

$$
\left\{\begin{array}{l}
10 \beta s+\alpha \geqslant 0, \\
20 \beta s^{2}+5 \alpha s+2 e \geqslant 0, \\
10 \beta s^{3}+4 \alpha s^{2}+(d+2 e) s+c \geqslant 0 \\
10 \beta s^{2}+3 \alpha s+d \geqslant 0, \\
10 \beta s^{3}+4 \alpha s^{2}+(d+2 e) s+c \geqslant 0 .
\end{array}\right.
$$

而 $y \in \mathbb{R}_{+}$, 所以当 $r>s_{2}, s>s_{2}$ 时, 有 $C_{r, s} \geqslant 0, D_{r, s} \geqslant 0$.

综上取 $r_{0}=s_{0}=\max \left\{r_{1}, s_{1}, s_{2}\right\}$, 当 $r>r_{0}, s>s_{0}, r$ 和 $s$ 为实数时, 有

$$
A_{r, s} \geqslant 0, \quad B_{r, s} \geqslant 0, \quad C_{r, s} \geqslant 0, \quad D_{r, s} \geqslant 0, \quad E_{r, s} \geqslant 0, \quad H_{r, s} \geqslant 0 .
$$

加上 $t \in \mathbb{R}_{+}$, 故此时有 $f_{r, s}(t) \geqslant 0$.

(II) 接下来讨论情形 $[\beta=\alpha=0, d+e>0, d \geqslant 0]$.

由条件 $d+e>0$, 可设 $e=h-d$, 其中 $h>0$, 代入 $f_{r, s}(t)$ 并整理为

$$
\begin{aligned}
& f_{r, s}(t)=d s r t(r t+s)(t-1)^{2}+A_{r, s}^{(1)} t^{5}+B_{r, s}^{(1)} t^{4}+C_{r, s}^{(1)} t^{3}+D_{r, s}^{(1)} t^{2}+E_{r, s}^{(1)} t+H_{r, s}^{(1)}, \\
& A_{r, s}^{(1)}=r\left(h r^{2}+(b+c) r+a\right), \quad H_{r, s}^{(1)}=s\left(h s^{2}+(b+c) s+a\right), \\
& B_{r, s}^{(1)}=r s(h r+b), \quad E_{r, s}^{(1)}=r s(h s+c), \\
& C_{r, s}^{(1)}=r s(2 h r+c), \quad D_{r, s}^{(1)}=r s(2 h s+b) .
\end{aligned}
$$

由引理 3.2 知存在正实数 $r_{0}, s_{0}$ 当 $r>r_{0}$ 和 $s>s_{0}$ 时,

$$
A_{r, s}^{(1)} \geqslant 0, \quad B_{r, s}^{(1)} \geqslant 0, \quad C_{r, s}^{(1)} \geqslant 0, \quad D_{r, s}^{(1)} \geqslant 0, \quad E_{r, s}^{(1)} \geqslant 0, \quad H_{r, s}^{(1)} \geqslant 0 .
$$

又显然 $\operatorname{dsrt}(r t+s)(t-1)^{2} \geqslant 0$, 故当 $r>r_{0}, s>s_{0}, r$ 和 $s$ 为实数时, 有 $f_{r, s}(t) \geqslant 0$.

(III) 再讨论情形 $[\beta=\alpha=d+e=0, d>0, b+c>0]$.

设 $h=b+c>0$, 即 $b=h-c$, 又 $e=-d$, 代入 $f_{r, s}(t)$ 并整理为

$$
f_{r, s}(t)=r s t(t-1)^{2}[(d r-c) t+d s-c]+\left(h r^{2}+a r\right) t^{5}+h r s t^{4}+h r s t+\left(h s^{2}+a s\right) .
$$

由引理 3.2 知存在正实数 $r_{0}$, 和 $s_{0}$, 当 $r>r_{0}, s>s_{0}$ 时,

$$
(d r-c) \geqslant 0, \quad(d s-c) \geqslant 0, \quad\left(h r^{2}+a r\right) \geqslant 0, \quad h r s \geqslant 0, \quad\left(h s^{2}+a s\right) \geqslant 0 .
$$

故当 $r>r_{0}, s>s_{0}, r$ 和 $s$ 为实数有 $f_{r, s}(t) \geqslant 0$.

(IV) 最后对剩下的情形 (引理 3.1 结论中最后 3 种情形) 类似讨论, 即可完成引理的证 明.

引理 3.4 二变元多项式 $f(x, u)$ 满足: $u$ 取正整数, 当 $x \in \mathbb{R}_{+}$时, 总有 $f(x, u) \geqslant 0$, 则 必存在正实数 $u_{0}$, 当 $u$ 取实数且 $u \geqslant u_{0}, x \in \mathbb{R}_{+}$时仍有 $f(x, u) \geqslant 0$.

证明 将 $f(x, u)$ 看作关于 $x$ 的单变元多项式, $u$ 视为参数.

$$
f(x, u)=p_{n}(u) x^{n}+p_{n-1}(u) x^{n-1}+\cdots+p_{0}(u),
$$


其中 $p_{n}(u)$ 不为零多项式. 我们有如下断言:

存在实数 $u_{1}$, 当 $u$ 取实数且 $u>u_{1}$ 时, $p_{n}(u)>0$.

这是因为 $p_{n}(u)$ 不为零多项式, 故当 $u$ 充分大时必恒有 $p_{n}(u)>0$ 或 $p_{n}(u)<0$, 但是当 $u$ 充分大取整数时, 要满足 $x \in \mathbb{R}_{+}$总有 $f(x, u) \geqslant 0$, 即 $f(x, u)$ 的首项系数不能小于 0 , 故必 有 $p_{n}(u)>0$.

又令 $\Delta\left(f\left(x^{2}, u\right), x\right)$ 是多项式 $f\left(x^{2}, u\right)$ 关于变元 $x$ 的判别式, 显见 $\Delta\left(f\left(x^{2}, u\right), x\right)$ 是关于 $u$ 的单变元多项式 (可能是 0 多项式). 当 $u$ 充分大时, $\Delta\left(f\left(x^{2}, u\right), x\right)$ 的值与它关于 $u$ 的最高 次项系数保持同号, 即存在正常数 $u_{2}$, 当 $u \geqslant u_{2}$ 时, $\Delta\left(f\left(x^{2}, u\right), x\right)$ 的符号保持不变 (其符号 恒为 “+” “-”或 “ 0 ”).

取 $u_{0}=\max \left\{u_{1}, u_{2}\right\}$, 则意味着参数 $u$ 在区间 $\left(u_{0},+\infty\right)$ 上连续变化时 $f\left(x^{2}, u\right)$ 看作 $x$ 的 多项式, 其实根个数和重数不变 (因为单变元多项式方程实根个数与重数只有在参数连续变 化跨过首项或判别式的实根时才可能发生变化, 见文献 [14] 118 页第 5-7 行). 而已知

$$
f\left(x^{2},\left[u_{0}\right]+1\right) \geqslant 0,
$$

故方程 $f\left(x^{2},\left[u_{0}\right]+1\right)=0$ 无奇数重实根, 也即: 方程 $f\left(x^{2}, u\right)=0$, 当 $u \geqslant u_{0}$ 时无奇数重实 根, 前已证 $p_{n}(u)>0$, 故

$$
f\left(x^{2}, u\right) \geqslant 0\left(u \geqslant u_{0}\right)
$$

即

$$
f(x, u) \geqslant 0, \quad\left(u \geqslant u_{0}, x \in \mathbb{R}_{+}\right)
$$

推论 $3.5 f\left(x_{1}, x_{2}, \ldots, x_{n}\right) \in S_{n, 5}$, 其中 $\left(x_{1}, x_{2}, \ldots, x_{n}\right) \in \mathbb{R}_{+}^{n}$. 若不等式 $f\left(x_{1}, x_{2}, \ldots\right.$, $\left.x_{n}\right) \geqslant 0$ 对一切自然数 $n$ 均成立, 则对每一个固定的非负整数 $\tilde{r}$, 必存在实数 $s_{0}(\tilde{r})$, 当 $s$ 取实 数且 $s>s_{0}(\tilde{r}), t \in \mathbb{R}_{+}$时, 有 $f_{\tilde{r}, s}(t) \geqslant 0$.

证明 对每一个固定的非负整数 $\tilde{r}, f_{\tilde{r}, s}(t)$ 可以看作关于 $t$ 和 $s$ 的二变元多项式 (见 (5) 式), 由引理 2.6 对任意自然数 $s$ 和实数 $t \in \mathbb{R}_{+}$都有 $f_{\tilde{r}, s}(t) \geqslant 0$, 故满足引理 3.4 的条件. 推论 的结论成立. 证毕.

推论 3.5 中的 $s_{0}(\tilde{r})$ 可以用引理 3.2 来有效的估计. 这样从引理 3.3 和推论 3.5 以及引 理 2.6 便获得了如下算法的正确性. 另外, 由于 $r$ 和 $s$ 具有完全对等的地位 (见 (7) 式), 针对 自然数变量 $s$ 的与 N3,N4 和 N5 相类似的步骤已省去.

\section{算法 nprove}

输入: 5 次有理系数对称型 $f\left(x_{1}, x_{2}, \ldots, x_{n}\right)=(n,[a, b, c, d, e, \alpha, \beta])$.

本算法判定在 $\left(x_{1}, x_{2}, \ldots, x_{n}\right) \in \mathbb{R}_{+}^{n}$ 上, 不等式 $f\left(x_{1}, x_{2}, \ldots, x_{n}\right) \geqslant 0$ 是否对一切自然数 $n$ 均成立, 成立则输出 “true”, 否则输出 “false”.

N1: 计算 $f_{r, s}(t)$ 的表达式 (6).

$\mathrm{N} 2$ : 计算引理 3.3 中的 $r_{0}$ 和 $s_{0}$, 记区域

$$
D \stackrel{\text { def }}{=}\left\{(r, s) \mid(r, s) \in \mathbb{R}_{+}^{2}, r \geqslant 1+\left[r_{0}\right], s \geqslant 1+\left[s_{0}\right]\right\} .
$$

$\mathrm{N} 21$ : 如果在 $D$ 上 $f_{r, s}(t) \geqslant 0\left(\forall t \in \mathbb{R}_{+}\right)$不成立, 输出 “false” 并停机. 
N3: 依次取 $\tilde{r}=1,2, \ldots,\left[r_{0}\right]$, 计算推论 3.5 中的 $s_{0}(\tilde{r})$. 记射线 $D \tilde{r} \stackrel{\text { def }}{=}\left\{s \mid s \in \mathbb{R}_{+}, s \geqslant\right.$ $\left.1+\left[s_{0}(\tilde{r})\right]\right\}$.

$\mathrm{N} 31$ : 如果在 $D \tilde{r}$ 上 $f_{\tilde{r}, s}(t) \geqslant 0\left(\forall t \in \mathbb{R}_{+}\right)$不成立, 输出 “false” 并停机.

否则继续以下步骤

N4: 记离散点集

$$
L \stackrel{\text { def }}{=} \bigcup_{\tilde{r}=1}^{\left[r_{0}\right]} L \operatorname{set}[\tilde{r}]
$$

其中 $L \operatorname{set}[\tilde{r}] \stackrel{\text { def }}{=}\left\{(\tilde{r}, 1), \ldots,\left(\tilde{r}, s_{0}(\tilde{r})\right)\right\}$.

N5: 如果 $(r, s) \in L, f_{r, s}(t) \geqslant 0\left(\forall t \in \mathbb{R}_{+}\right)$不成立, 则输出 “false” 并停机. 否则输出 “true” 并停机.

算法步骤 N21, N31, N5 中的选择判定均已在 Tarski 模型内, 故可以用证明代数不等式的 胞腔分解算法来完成, 目前已经有好几个软件可以做胞腔分解, 如 REDLOG ${ }^{[15]}, \mathrm{QEPCAD}^{[10]}$, DISCOVERER $^{[16]}$, BOTTEMA ${ }^{[17,18]}$, 其中以 BOTTEMA 最为适合我们的编程需要. 另外, 因 使用引理 3.2 来得引理 3.3 中 $r$ 和 $s$ 的界比较粗, 在具体编程实现时, 我们做了一些变通, 使 用了 BOTTEMA 中的 xmin 命令来计算 $r$ 和 $s$ 的最佳的界.

上述算法显然是可停机的. 这样我们就证明了定理 1.

\section{4 一些实例}

例 1

$$
\begin{aligned}
f= & -\left(\sum_{k=1}^{n} x_{k}^{5}\right)-6\left(\sum_{k=1}^{n} x_{k}^{4}\right)\left(\sum_{k=1}^{n} x_{k}\right) \\
& +2\left(\sum_{k=1}^{n} x_{k}^{3}\right)\left(\sum_{k=1}^{n} x_{k}^{2}\right)+8\left(\sum_{k=1}^{n} x_{k}^{3}\right)\left(\sum_{k=1}^{n} x_{k}\right)^{2} \\
& +3\left(\sum_{k=1}^{n} x_{k}^{2}\right)^{2}\left(\sum_{k=1}^{n} x_{k}\right)-6\left(\sum_{k=1}^{n} x_{k}^{2}\right)\left(\sum_{k=1}^{n} x_{k}\right)^{3}+\left(\sum_{k=1}^{n} x_{k}\right)^{5},
\end{aligned}
$$

其中 $x_{i} \in \mathbb{R}_{+}, i=1, \ldots, n$. 请问是否对一切自然数 $n$ 均有 $f \geqslant 0$ 成立?

这是我们前面谈到的问题 1. 利用程序 nprove, 只需在 Maple 环境下键入命令 “nprove ([-1,-6,2,8,3,-6,1]); ”, 机器运行 30.7 秒 (不同的机器配置得出的测试时间可能不同, 我们使用 的处理器是 Pentium IV, $2.4 \mathrm{G})$ 后输出 “true”. 故对一切自然数 $n, \forall x_{i} \in \mathbb{R}_{+}(i=1, \ldots, n)$ 均 有 $f \geqslant 0$ 成立.

例 2

$$
\begin{aligned}
f= & -7\left(\sum_{k=1}^{n} x_{k}^{4}\right)+8\left(\sum_{k=1}^{n} x_{k}^{3}\right)\left(\sum_{k=1}^{n} x_{k}\right) \\
& +4\left(\sum_{k=1}^{n} x_{k}^{2}\right)^{2}-6\left(\sum_{k=1}^{n} x_{k}^{2}\right)\left(\sum_{k=1}^{n} x_{k}\right)^{2}+\left(\sum_{k=1}^{n} x_{k}\right)^{4},
\end{aligned}
$$

其中 $x_{i} \in \mathbb{R}_{+}, i=1, \ldots, n$. 请问是否对一切自然数 $n$ 均有 $f \geqslant 0$ 成立? 
此问题按引理 2.5 来做, 用时 523.5 秒, 可证得当 $n \leqslant 10000000$ 时 $f \geqslant 0$ 成立. 而用程序 nprove, 键入命令 “nprove $([-7,8,4,-6,1])$; ”, 机器运行 5.48 秒后输出 “true”, 于是证得了对一 切自然数 $n$ 均有 $f \geqslant 0$ 成立. 由此可见二者之间的差别.

例 3

$$
\begin{aligned}
f= & -\left(\sum_{k=1}^{n} x_{k}^{5}\right)-6\left(\sum_{k=1}^{n} x_{k}^{4}\right)\left(\sum_{k=1}^{n} x_{k}\right) \\
& +195\left(\sum_{k=1}^{n} x_{k}^{3}\right)\left(\sum_{k=1}^{n} x_{k}^{2}\right)-11\left(\sum_{k=1}^{n} x_{k}^{3}\right)\left(\sum_{k=1}^{n} x_{k}\right)^{2} \\
& -2\left(\sum_{k=1}^{n} x_{k}^{2}\right)^{2}\left(\sum_{k=1}^{n} x_{k}\right)-9\left(\sum_{k=1}^{n} x_{k}^{2}\right)\left(\sum_{k=1}^{n} x_{k}\right)^{3}+\left(\sum_{k=1}^{n} x_{k}\right)^{5},
\end{aligned}
$$

其中 $x_{i} \in \mathbb{R}_{+}, i=1, \ldots, n$. 请问是否对一切自然数 $n$ 均有 $f \geqslant 0$ 成立?

在 Maple 环境键入指令 “nprove $([-1,-6,195,-11,-2,-9,1])$ ”, 用时 145 秒, 输出结果为 “false”. 故不等式 $f \geqslant 0$ 不是对一切自然数 $n$ 普遍成立. 事实上这个不等式对 $n \leqslant 6$ 成立, 对 $n \geqslant 7$ 不成立.

\section{5 结束语}

本文中讨论的一类正性判定问题已在 Tarski 模型之外. 这一工作是在吴文俊倡导的数 学机械化思想下所做的新的尝试, 这种尝试只是开了一个头, 还有许多工作需要做.

定理 1 只部分地回答了问题 2 , 即 5 次以下对称型的正性是可判定的. 我们猜测整个问 题 2 的答案都应当是肯定的, 即任意次数的对称型的正性都是可判定的. 证明的困难之处在 于多变元多项式类似于引理 3.4 的结论一般并不成立. 经简单的极限过程还可以得到相应类 型无穷级数不等式的机器判定算法. 另外, 我们觉得还有以下几个问题值得进一步探讨:

(i) 对称型 $g\left(x_{1}, \ldots, x_{n}\right)$ 的系数不是常系数, 而是具有与 $n$ 相关的变系数时, 问题 2 的答 案如何?

例

$$
\begin{aligned}
f= & n(n-1)\left(\sum_{k=1}^{n} x_{k}^{5}\right)-(n+6)(n-1)\left(\sum_{k=1}^{n} x_{k}^{4}\right)\left(\sum_{k=1}^{n} x_{k}\right) \\
& -\left(2 n^{2}-4 n+4\right)\left(\sum_{k=1}^{n} x_{k}^{3}\right)\left(\sum_{k=1}^{n} x_{k}^{2}\right)+(6 n-4)\left(\sum_{k=1}^{n} x_{k}^{3}\right)\left(\sum_{k=1}^{n} x_{k}\right)^{2} \\
& +\left(n^{2}-n+3\right)\left(\sum_{k=1}^{n} x_{k}^{2}\right)^{2}\left(\sum_{k=1}^{n} x_{k}\right)-(2 n+2)\left(\sum_{k=1}^{n} x_{k}^{2}\right)\left(\sum_{k=1}^{n} x_{k}\right)^{3}+\left(\sum_{k=1}^{n} x_{k}\right)^{5},
\end{aligned}
$$

其中 $x_{i} \in \mathbb{R}_{+}, i=1, \ldots, n$. 请问是否对一切自然数 $n$, 不等式 $f \geqslant 0$ 均成立?

(ii) 对称型 $g\left(x_{1}, \ldots, x_{n}\right)$ 中变元的变化范围由 $\mathbb{R}_{+}^{n}$ 改为 $\mathbb{R}^{n}$, 问题 2 的答案又将如何?

(iii) 对于 6 次 (或更高次) 的 $n$ 个变元的对称型, 其半正定性是否可以判定?

致谢 非常感谢审稿人提出的有益建议. 


\section{参考文献}

1 Tarski A. A Decision method for Elementary Algebra and Geometry. 2nd ed. Berkeldy: University of California Press, 1951

2 吴文俊. 初等几何判定问题与机械化证明. 中国科学, 1977, 20(6): 507-516

3 Chou S C. Mechanical Geometry Theorem Proving. Dordrecht: Reidel, 1988

4 Buchberger B. Grobner bases: An algorithmic method in polynomial ideal theory. In: Multidimensional Systems Theory. Dordrecht: Reidel, 1985, 184-232

5 Kapur D. Geometry theorem proving using Hilbert's Nullstellensata. In: Proc SYMSAC'86, New York: ACM Press, 1986, 202-208

6 Kutzler B, Stifter S. Automated geometry theorem proving using Buchberger's algorithm. In: Proc SYMSAC'86, New York: ACM Press, 1986, 209-214

7 杨路, 张景中, 侯晓荣. 非线性代数方程组与定理机器证明. 上海: 上海科技教育出版社, 1996

8 Zhang J Z, Yang L, Deng M K. The parallel numerical method of mechanical theorem proving. Theor Comput Sci, 1990, 74(3): 253-271

9 Chou S C, Zhang J Z, Gao X S. Machine Proofs in Geometry: Automated Production of Readable Proofs for Geometry Theorems. Singapore: World Scientific, 1994

10 Collins G E, Hong H. Partial cylindrical algebraic decomposition for quantifier elimination. Journal of Symbolic Computation, 1991, 12: 299-328

11 Timofte V. On the positivity of symmetric polynomial functions I. J Math Anal Appl, 2003, 284: 174-190

12 Timofte V. On the positivity of symmetric polynomial functions. Part II: Lattice general results and positivity criteria for degrees 4 and 5. J Math Anal Appl, 2005, 284: 652-667

13 Choi M D, Lam T Y, Reznick B. Even symmetric sextics. Math Z, 1987, 195: 559-580

14 杨路, 夏壁灿. 计算实代数几何. 王东明等主编. 符号计算选讲. 北京: 清华大学出版社, 2003, 110-111

15 Dolzman A, Sturm T. REDLOG: Computer algebra meets computer logic. ACM SIGSAM Bulletin, 1997, 31(2): 2-9

16 Yang L, Hou X R, Xia B C. A complete algorithm for automated discovering of a class of inequality-type theorems. Sciences in China, Ser F, 2001, 44: 33-49

17 杨路, 夏时洪. 一类构造性几何不等式的机器证明. 计算机学报, 2003, 26(7): 769-778

18 Yang L, Zhang J. A practical program of automated proving for a class of geometric inequalities. In: Automated Deduction in Geometry, Lecture Notes in Artificial Intelligence Vol 2061. Berlin Heidelberg: Springer-Verlag, 2001. 41-57 